\title{
Fluxnet Synthesis Dataset Collaboration Infrastructure
}

\author{
Deb Agarwal (LBNL), Marty Humphrey (UVa), Catharine van Ingen (Microsoft), \\ Norm Beekwilder (UVa), Monte Goode (LBNL), Keith Jackson (LBNL), Matt \\ Rodriguez (LBNL), and Robin Weber(UCB)
}

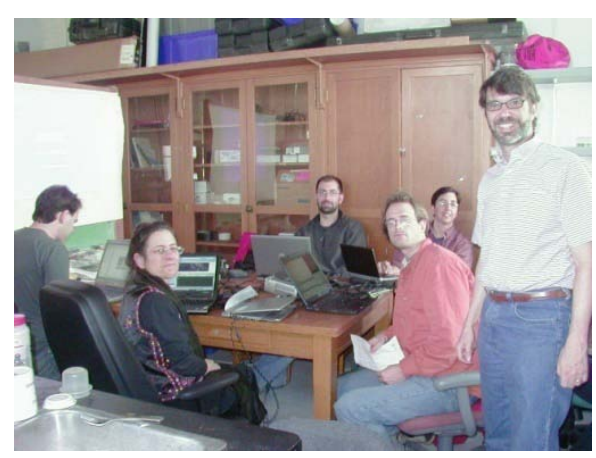

The Fluxnet synthesis dataset originally compiled for the La Thuile workshop contained approximately 600 site years. Since the workshop, several additional site years have been added and the dataset now contains over 920 site years from over 240 sites. A data refresh update is expected to increase those numbers in the next few months. The ancillary data describing the sites continues to evolve as well. There are on the order of 120 site contacts and 60 proposals have been approved to use the data. These proposals involve around 120 researchers. The size and complexity of the dataset and collaboration has led to a new approach to providing access to the data and collaboration support and the support team attended the workshop and worked closely with the attendees and the Fluxnet project office to define the requirements for the support infrastructure.

As a result of this effort, a new web site (http://www.fluxdata.org) has been created to provide access to the Fluxnet synthesis dataset. This new web site is based on a scientific data server which enables browsing of the data on-line, data download, and version tracking. We leverage database and data analysis tools such as OLAP data cubes and web reports to enable browser and Excel pivot table access to the data. The data cubes provide organization and aggregation of data along dimensions such as time to
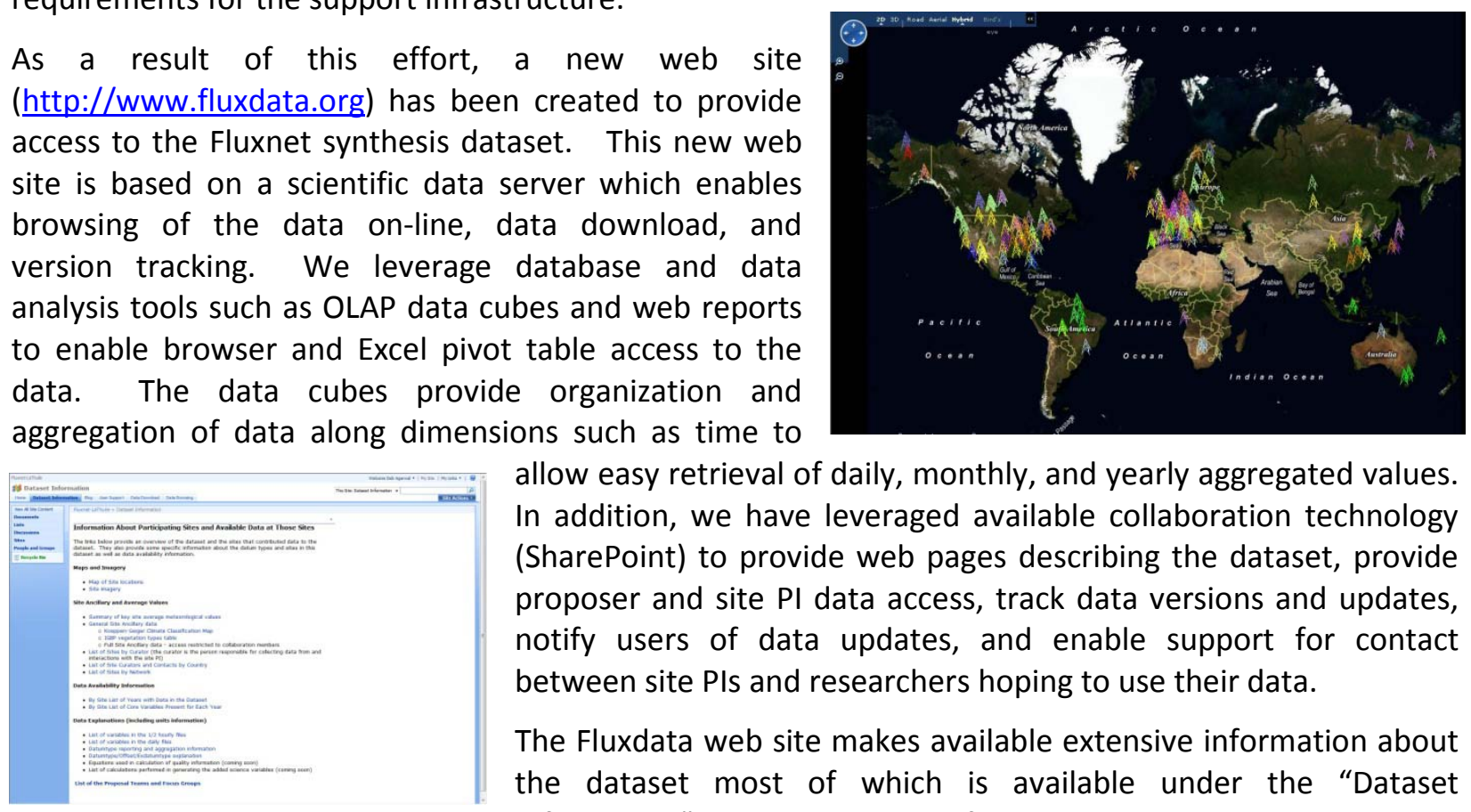

allow easy retrieval of daily, monthly, and yearly aggregated values. In addition, we have leveraged available collaboration technology (SharePoint) to provide web pages describing the dataset, provide proposer and site PI data access, track data versions and updates, notify users of data updates, and enable support for contact between site PIs and researchers hoping to use their data.

The Fluxdata web site makes available extensive information about the dataset most of which is available under the "Dataset Information" tab. Here you will find interactive site maps, basic site

information, average values, and data availability information as well as descriptions and explanations of the dataset variables and data levels. These reports are presented as spreadsheets for ease of download and are derived from a database containing the Fluxnet data and are refreshed periodically. A list of the approved proposals along with the coordinator and collaborators on the proposal as well as the sites planned for use in the analysis. Links to network specific information are also provided. Currently

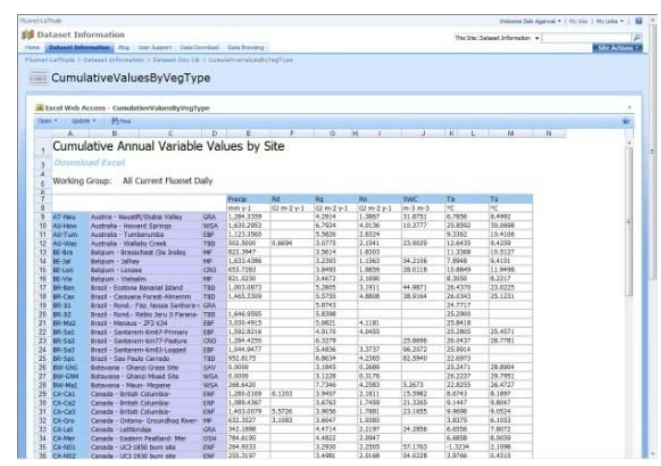


only the AmeriFlux network has information posted but other networks are welcome to contribute

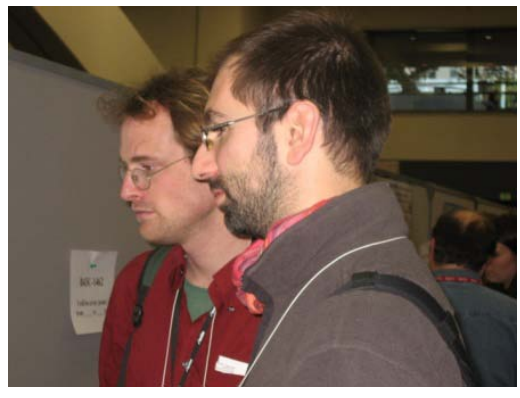
information as well.

The "Blog" contains news items about the web site, dataset, and processing progress. The "User Support" tab provides access to a user manual describing how to use each of the areas of the site.

Authorized members of the Fluxnet collaboration can sign-in at the web site and obtain access to a range of additional functions. The "Data Browsing" tab provides access to all available site ancillary data as well as summary annual values for the variables. It also provides direct connection to the data cube for direct browsing of the data. The "Data Download" tab enables download of site years selected by the user. Changes to site ancillary information can also be submitted via the "Ancillary Data Update" tab. Proposal coordinators are able to maintain the list of sites in use for their analysis and communicate with site PIs via e-mail. The next major functionality planned for the Fluxdata web site is to add site PI access to flux-met and ancillary data from their site and support for communication with proposal teams using the site.

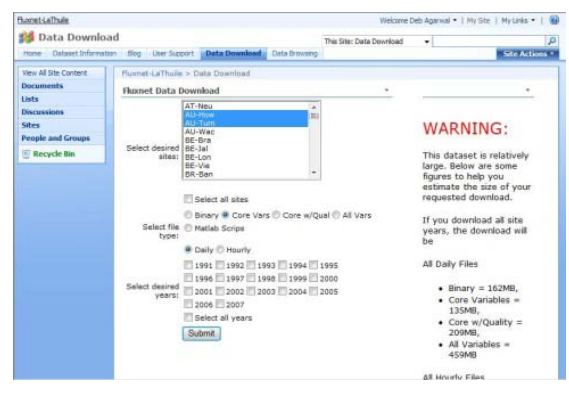

Using the Fluxdata web site and Scientific Data Server, researchers can keep up-to-date with the latest

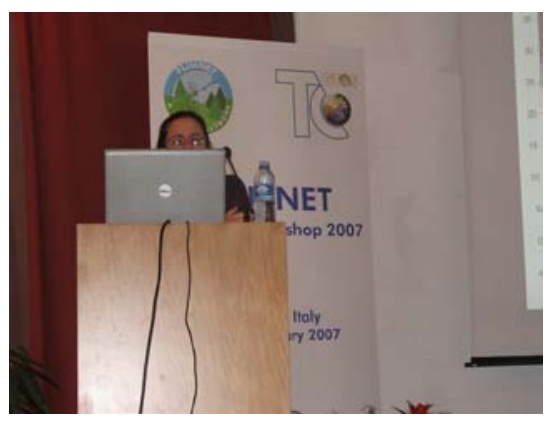
updates to the dataset, select sites of interest for analysis, and communicate with others in the collaboration. The resulting site and capabilities we believe allow individual researchers to concentrate on science rather than data management. This site and server are brought to you by the Berkeley Water Center and University of Virginia in collaboration with the Fluxnet community and in particular Dario Papale. This site is continually evolving and we welcome comments and input from the community. You can reach the support team at fluxdata-support@fluxdata.org.

The research and development described in this paper is funded by Microsoft Research's eScience program. This work is also supported by the Director, Office of Science, of the U.S. Department of Energy under Contract No. DE-AC02-05CH11231.

This article appeared in the FLUXNET newsletter: FluxLetter, Vol 1, Issue 1, 2008, pp. 5-7. 